\title{
An Improved PSO Algorithm Based on Mutation Operator and Simulated Annealing
}

\author{
Xiaojun Deng, Zhiqiang Wen, Yu Wang and Pingan Xiang \\ College of Computer and Communication, Hunan University of Technology, \\ Zhuzhou 412007, China
}

\begin{abstract}
Particle swarm optimization (PSO) algorithm is simple stochastic global optimization technique, but it exists unbalanced global and local search ability, slow convergence speed and solving accuracy. An improved simulated annealing (ISAM) algorithm is introduced into the PSO algorithm with crossover and Gauss mutation to propose an improved PSO (ISAMPSO) algorithm based on the mutation operator and simulated annealing in this paper. In the ISAMPSO algorithm, the mutation operator of genetic algorithm is introduced into the SA algorithm as a generation mechanism of new solution in order to propose an improved simulated annealing algorithm with mutation (ISAM). Then the ISAM algorithm is introduced into the PSO algorithm to jump out the local optimum, effectively achieve the global optimum adjust and optimize the population, maintain the diversity of the population, improve the local search ability and convergence speed. Six classical functions are selected to test the performance of the proposed ISAMPSO algorithm. The simulation experiments results show that the proposed ISAMPSO algorithm can effectively overcomes the stagnation phenomenon and enhance the global search ability. The convergence speed and accuracy were better than the PSO algorithm.
\end{abstract}

Keywords: Particle swarm optimization; mutation operator; simulated annealing algorithm; optimization; classical function

\section{Introduction}

Particle swarm optimization (PSO) [1] algorithm was introduced by Kennedy and Eberhar in 1995. The PSO algorithm is a population-based search algorithm based on the simulation of the social behavior of birds or fishes within a flock. It takes on these characteristics of the easy describing and realization, the rarely adjusting parameters, the relatively smaller population, less number of function evaluations, fast convergence speed, parallel processing and robustness. It can find the global optimal solution of the solving problem with larger probability, and the calculation efficiency is higher than traditional random methods. The PSO algorithm has profound intelligent background, and is suitable for scientific research and engineering application) [2]. Therefore, the PSO algorithm has been aroused widespread concern in evolutionary computation research field. And a large number of research results are obtained in a few years. It has been successfully applied in function optimization, neural network training, fuzzy control system, classification, pattern recognition, signal processing, robot technology and so on [3-5].

Compared to other evolutionary algorithms, the PSO algorithm has great advantages in solving the problem of high dimension and complex. However, the PSO algorithm likes other intelligent optimization algorithms, it is also easy to fall in the local extreme points, slow convergence speed in the later evolution, lost diversity and poor optimization accuracy. So many researchers have proposed a large number of improved algorithms, which improve the performance of the basic PSO algorithm on some extent. Shi et al. [6] 
presented a variable population-size genetic algorithm (VPGA) by introducing the "dying probability" for the individuals and the "war/disease process" for the population. Tang et al. [7] presented a simulated annealing particle swarm optimization algorithm (SA-PSO) to solve the complicated and nonlinear generation expansion planning of hydro-thermal power system (include nuclear plant). Kim and Hirota [8] presented a genetic algorithmparticle swarm optimization (GA-PSO) algorithm based on vector control for loss minimization operation of induction motor. Li et al. [9] presented a new evolutionary learning algorithm based on a hybrid of improved real-code genetic algorithm (IGA) and PSO algorithm, called HIGAPSO algorithm. Niknam et al. [10] presented a hybrid evolutionary programming based clustering algorithm, called PSO-SA, by combining PSO and SA. Neoh et al. [11] presented a layered encoding cascade evolutionary approach to solve a 0/1 knapsack optimization problem, and a layered encoding structure based on the schema theorem and the concepts of cascade correlation and multipopulation evolutionary algorithms. Behnamian and Fatemi Ghomi [12] presented a PSOSA hybrid metaheuristic based on combining the advantages of PSO and SA for a new comprehensive regression model to time-series forecasting. Lee and Hsiao [13] adopted the S-system model to represent the gene network and esTablelish a methodology to infer the model. And an adaptive genetic algorithm-particle swarm optimization hybrid method is proposed to infer appropriate network parameters. Thanushkodi and Deeba [14] presented a variant of PSO, which is hybridized with the simulated annealing approach to achieve better solutions. Rahim et al. [15] presented a hybrid particle swarm and genetic algorithm based on discrete augmented Lagrangian approach to achieve the optimal value of Lagrange multipliers and required parameters. Soleymani [16] presented a new method that uses the combination of PSO and SA to predict the bidding strategy of Generating Companies (Gencos) in an electricity market where they have incomplete information about their opponents and market mechanism of payment is pay as bid. Tajbakhsh et al. [17] presented a very fast hybrid metaheuristic algorithm based on combining PSO and SA. Wang et al. [18] presented a simulated annealing particle swarm (PSO-SA) algorithm for solving the shortest path on curved surface. Lee and Ponnambalam [19] presented two evolutionary heuristics to determine the cutting parameters in multipass turning operations. Sheikhalishahi et al. [20] presented a novel hybrid GA- PSO approach for reliability redundancy allocation problem (RRAP) in series, series-parallel, and complex (bridge) systems. Galvez and Iglesias [21] presented a novel hybrid evolutionary approach (called IMCHGAPSO) for B-spline curve reconstruction comprised of two classical bio-inspired techniques: GA and PSO, accounting for data parameterization and knot placement, respectively. Pan and Das [22] presented a new hybrid regrouping PSOGA approach to design sub-optimal state feedback regulators over networked control systems with random packet losses. Rabbani et al. [23] presented a new hybrid GA-PSO metaheuristic algorithm to be applicable and reliable comparing its numerical results with GAMS. Wang et al. [24] presented a new PSO-SA algorithm, which focuses on researching the effect of inertia weight of PSO-SA algorithm on the performance of the algorithm. Khoshahval et al. [25] presented a new parallel optimization (P-PSOSA) algorithm for performing the fuel management optimization. Two different fitness function considering the multiplication factor maximizing and power peaking factor minimizing objectives are simultaneously defined. Martínez-Soto et al. [26] presented a hybrid PSO-GA optimization method for automatic design of fuzzy logic controllers (FLC) to minimize the steady state error of a plant's response. Sahoo et al. [27] presented an efficient hybrid approach based on genetic algorithm and particle swarm optimization for solving mixed integer nonlinear reliability optimization problems in series, seriesparallel and bridge systems. Sarasvathi et al. [28] presented a hybrid PSO-GA based on integrating the strength of PSO and GA to find the routing with QoS satisfied and an interference free path from the redundant paths. Tao et al. [29] presented an improved particle swarm algorithm (SA-PSO) to solve this problem, which can restrict the position 
change of original and new particles in the iteration process and accelerate the convergence speed of the algorithm.

The SA algorithm is a computational stochastic technique for obtaining near global optimum solutions to the complex optimization problems. It is inspired from the thermodynamic process of annealing of molten metals to attain the lowest free energy state. And the mutation operator can randomly change value of individual genes in order to take on the diversity of individuals in the population for guiding the search. So the idea of SA algorithm and mutation operator are introduced into the particle swarm optimization algorithm to propose an improved PSO(ISAMPSO) algorithm based on the mutation operator and simulated annealing in this paper. The ISAMPSO algorithm combines the global search optimization ability and simple realization of PSO algorithm with stronger jumping out of local optimal solution ability of SA algorithm and guiding the search ability of mutation operation in order to avoid falling into local extreme point of PSO algorithm and improve the convergence speed and accuracy of ISAMPSO algorithm in the late evolution.

The rest of this paper is organized as follows. Section 2 briefly introduces particle swarm optimization (PSO) algorithm. Section 3 briefly introduces mutation operator and simulated annealing (SA) algorithm. Section 4 presents an improved PSO (ISAMPSO) algorithm based mutation operator and simulated annealing (SA) algorithm. In this section, the idea and the steps of the ISAMPSO algorithm are introduced in detail. Section 5 applies the ISAMPSO algorithm for solving several benchmark functions. Finally, the conclusions are discussed in Section 6 .

\section{Particle swarm Optimization (PSO) Algorithm}

The PSO algorithm [1] is a population-based search algorithm based on the simulation of the social behavior of birds within a flock. In PSO algorithm, individuals, referred to as particles, are "flown" through hyper dimensional search space. The particles' positions within the search space are changed based on the social-psychological tendency of individuals in order to delete the success of other individuals. The changing of one particle within the swarm is influenced by the experience, or knowledge. The consequence of modeling for this social behavior is that the search is processed in order to return toward previously successful regions in the search space. Namely, the velocity $(v)$ and position $(x)$ of each particle will be changed by the particle best value $(p B)$ and global best value $(g B)$. The velocity and position updating of the particle is shown in the followed expression:

$$
\begin{aligned}
& v_{i j}(t+1)=w v_{i j}(t)+c_{1} r_{1}\left(p B_{i j}(t)-x_{i j}(t)\right)+c_{2} r_{2}\left(g B_{i j}(t)-x_{i j}(t)\right) \\
& x_{i j}(t+1)=x_{i j}(t)+v_{i j}(t+1)
\end{aligned}
$$

Where $v_{i j}(t+1)$, velocities of particle $i$ at iterations $j, x_{i j}(t+1)$, positions of particle $i^{\text {th }}$ at iterations $j^{\text {th }} . w$ is inertia weight to be employed to control the impact of the previous history of velocities. Accordingly, the parameter $w$ regulates the trade-off between the global and local exploration abilities of the swarm.

The basic flow chart of PSO algorithm is shown in Figure1. 


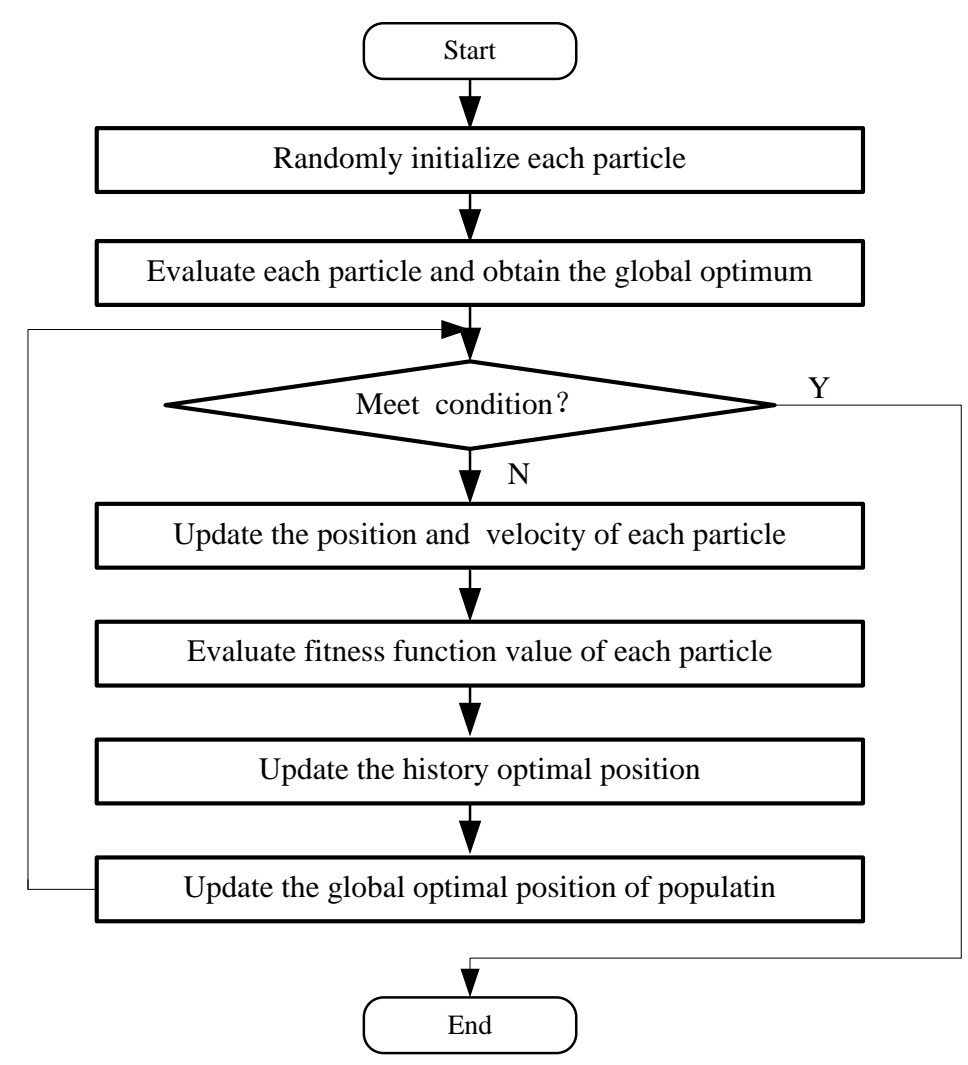

Figure 1. The Basic Flow Chart of PSO Algorithm

\section{Mutation Operator and Simulated Annealing (SA) Algorithm}

\subsection{Mutation Operator}

The crossover operation plays a vital role in genetic algorithm. But it only permutes and combines the existing genes, so new genes cannot be generated. With the further search, the individuals in the population have local similarity, which will result in the failure crossover, make the search in the stagnation state, fall into the local optimal solution, namely the premature phenomenon. At this time, the mutation operation is particularly important. The mutation operator can randomly change value of individual genes to take on the diversity of individuals in the population, which guide the search, and have the chance to climb the mountain and converge to the global optimum. So the mutation operation is the most important means to guide the search to climb mountain and prevent the convergence before the mature.

\subsection{Simulated Annealing (SA) Algorithm}

The basic idea of SA algorithm comes from the physical annealing principle [30]. The object particles move in disorder at high temperature, and the particles can converge to a lowest energy state when the temperature gradually decreases. The purpose of converging to the optimal solution is realized. First, the SA algorithm generates a random solution on the problem solution space, which is disturbed. Then the state transfer process of the solid particle under a certain temperature is simulated. The obtained solutions by disturbing is evaluated, and the solution is compared with the current solution and replaced according the Metropolis criterion. The SA algorithm repeats disturbing under the same temperature in order to simulate all kinds of energy states in the solid. In addition, the SA algorithm imitates the decreasing process of temperature by changing its parameters until the 
temperature reaches a given value. At this time, the obtained solution is regarded as the final solution, which is equivalent to the lowest energy state. When the SA algorithm is used to solve the optimization problem, the basic flow chart is shown in Figure2.

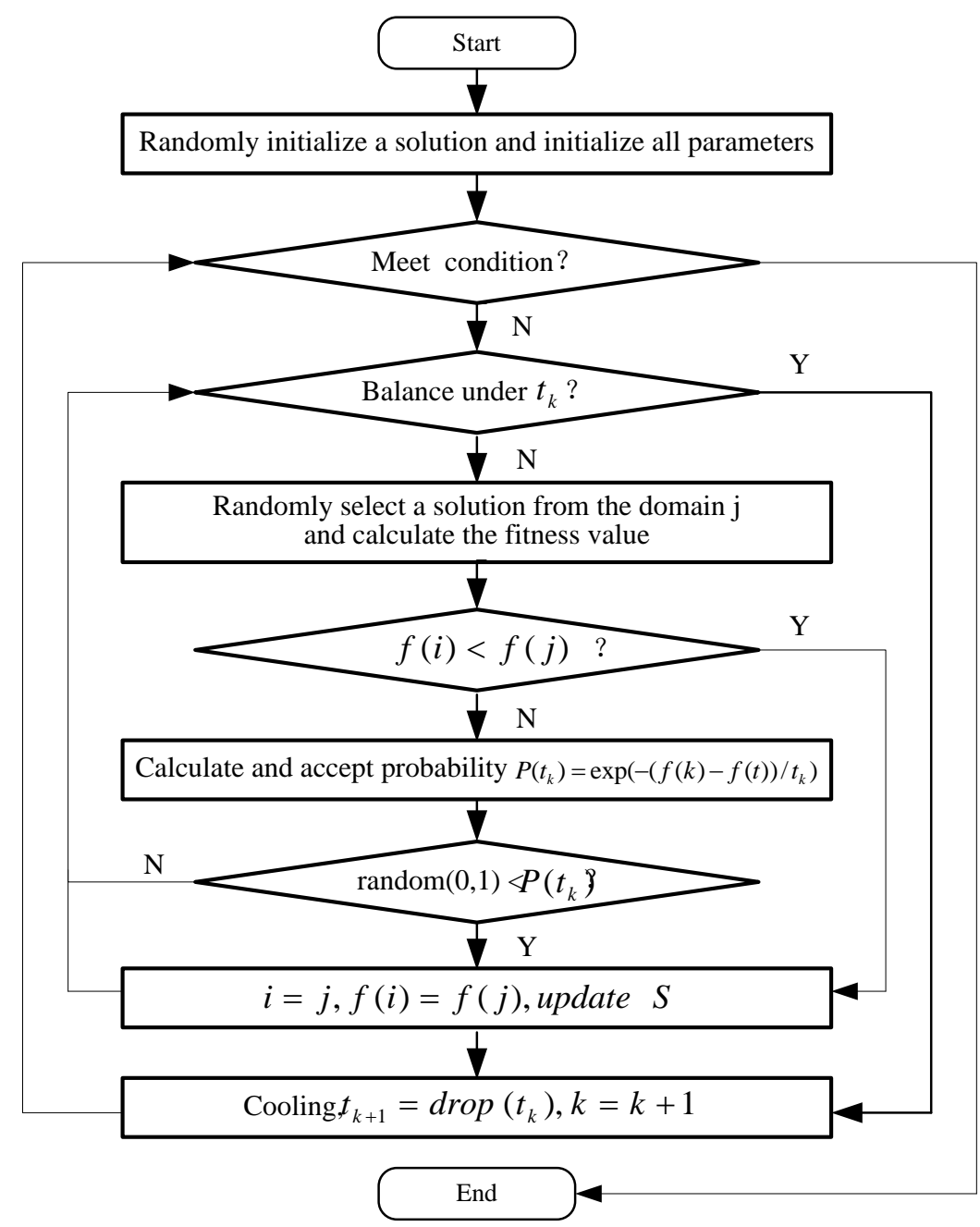

Figure 2. The Flow of the SA Algorithm

The core theory of the SA is to allow the occasional worsening moves, so that these can eventually help locate the neighborhood to the global minimum. The associated expression of the probability is given by the Boltzmann:

$$
\operatorname{probability}(p)=\exp \left(\frac{-\Delta E}{K_{b} T}\right)
$$

In the expression (3), $K_{b}$ is a constant, $\Delta E$ is the change in the energy value from one point to the next, $T$ is the temperature.

For the optimization the energy term, $\Delta E$ refers to the value of the e function, $\mathrm{T}$ is a control parameter that regulates the process of annealing. The acceptance criterion is popularly referred to as the Metropolis criterion. And the improving and deteriorating move of this acceptance criterion has been proposed by Galuber:

$$
\operatorname{probability}(p)=\frac{\exp (-\Delta E / T)}{1+\exp (-\Delta E / T)}
$$




\section{An Improved PSO (ISAMPSO) Algorithm}

The mutation operator is the most important methods to guide the search for climbing mountain and preventing the convergence. The SA algorithm unceasingly generates new solutions with solutions, and in accordance with the following criteria to determine whether to accept by using generation mechanism of new solution from an initial solution. And determine whether to accept a new solution according to Metropolis criterion. The generation of new solution is the most time-consuming step in SA algorithm, but it directly affects the search efficiency of solution space. The PSO algorithm based on mutation operator is often difficult to obtain the optimal solution with higher precision due to existing the visual and step randomness and random behavior. And the SA algorithm has the advantages of high quality, strong robustness of the initial value, the strong local search ability. However, it is suitable to combine different algorithms based on advantage complementary for finding a method to solve this problem. So an improved PSO (ISAMPSO) algorithm based on the satisfactory solution of fast found global extreme value of the mutation operator and strong local search ability of simulated annealing under low temperature in this paper. In the ISAMPSO algorithm, the mutation operator of genetic algorithm is introduced into the SA algorithm as a generation mechanism of new solution in order to propose an improved simulated annealing algorithm with mutation (ISAM). Then the ISAM algorithm is introduced into the PSO algorithm to jump out the local optimum, effectively achieve the global optimum adjust and optimize the population, maintain the diversity of the population, improve the local search ability and convergence speed. So the ISAMPSO algorithm takes on strong global search ability and local search ability.

The steps of the proposed ISAMPSO algorithm are obtained as follows:

Step1. Initialize

Randomly generate population( $m$ ), the velocity and position of each particle is initialized. Set the cognition learning factor $\left(c_{1}\right)$, social learning factor $\left(c_{2}\right)$,inertia weight $(\omega)$, the number of maximum iteration $\left(G_{\max }\right)$, initial acceptance probability $\left(P_{r}\right)$.

Step2. Calculate the fitness value

According to the objective optimization problem, the fitness value of each individual is calculated. The best position of particle is Pbest and global best of population is Gbest .

Step3. Initialize the annealing temperature

$$
T_{0}=\frac{f_{\text {min }}^{0}-f_{\text {max }}^{0}}{\ln P_{r}}=-\frac{|\Delta f|}{\ln P_{r}}
$$

Step4. Determine

Determine whether the termination condition is meet. If the termination condition is meeting, go to Step 11. Otherwise, go to Step 5.

Step5. Calculate the fitness of each particle $\left(f_{i}(k)\right)$ and the average fitness $\left(f_{\text {avgi }}(k)\right)$.

Step6. If the fitness of particle is better than Pbest, the current fitness is set as Pbest . The optimal individual extreme value is selected as Gbest .

Step7. Update the position and velocity of the particles

Step8. Calculate the fitness of each particle $\left(f_{i}(k+1)\right)$ and the average fitness $\left(f_{\text {avgi }}(k+1)\right)$.

Step9. The mutation operator is used to generate the new mutation.

Step10. Determine whether to accept a new solution.

Calculate the caused change amount of fitness value between two positions $\Delta f=f_{i}(k+1)-f_{i}(k)$. If $\Delta f<0$, a new solution is accepted. Otherwise it will remain the old solution.

Step11. Output the optimal solution. 


\section{Experimental Results and Analysis}

In order to test the optimization performance of the proposed ISAMPSO algorithm for solving complex problem, several benchmark functions (Rastrigrin, Schaffer's f6, Sphere, Rosenbrock, Griewank) are selected in this paper. The PSO algorithm and SAPSO algorithm are select to compare with the proposed ISAMPSO algorithm. The parameters of the algorithms are selected after testing. We started with some classic values that have already been used in other studies papers, and then we modified these values until the selected values are chosen. The selected ones are those that gave the best computational results concerning both the quality of the solution and the run time needed to achieve this solution. The parameters of three algorithms are given in Table.1. The experiment environment are: the Pentium IV, 2.0GB RAM, Matlab 2012b. Several benchmark functions are shown Table. 2.

Table 1. The Parameters of Three Algorithms

\begin{tabular}{lccc}
\hline \multicolumn{1}{c}{ Parameters } & PSO & SAPSO & ISAMPSO \\
\hline Population size $(m)$ & 60 & 60 & 60 \\
Iteration times $\left(G_{\max }\right)$ & 1000 & 1000 & 1000 \\
inertia weight $(w)$ & 0.80 & 0.80 & 0.80 \\
Max velocity $(v)$ & 100 & 100 & 100 \\
Cognition learning factor $\left(c_{1}\right)$ & $c_{1}=2$ & $c_{1}=2$ & $c_{1}=2$ \\
Social learning factor $\left(c_{2}\right)$ & $c_{2}=2$ & $c_{2}=2$ & $c_{2}=2$ \\
Initial temperature $\left(T_{0}\right)$ & N/A & 15 & 15 \\
Initial acceptance probability $\left(P_{r}\right)$ & N/A & $P_{r}=0.7$ & $P_{r}=0.7$ \\
Mutation probability $\left(P_{m}\right)$ & N/A & 0.1 & 0.1 \\
\hline
\end{tabular}

Table 2. Benchmark Functions

\begin{tabular}{ccccc}
\hline Function & Function Expression & Opt. & Dim. & Range \\
\hline Rastrigrin & $f_{1}(x)=\sum_{i=1}^{n}\left[x_{i}^{2}-10 \cos \left(2 \pi x_{i}\right)+10\right]$ & $\mathbf{0}$ & $\mathbf{3 0}$ & $(-10,10)^{n}$ \\
Schaffer's f6 & $f_{2}(x)=\frac{\left(\sin \left(\sqrt{x_{2}+y_{2}}\right)^{2}-0.5\right.}{\left(1.0+0.001\left(x^{2}+y^{2}\right)\right)^{2}}+\frac{1}{2}$ & $\mathbf{0}$ & $\mathbf{2}$ & $(-100,100)^{2}$ \\
Sphere & $f_{3}(x)=\sum_{i=1}^{n} x_{i}^{2}$ & $\mathbf{0}$ & $\mathbf{3 0}$ & $(-100,100)^{n}$ \\
Rosenbrock & $f_{4}(x)=\sum_{i=1}^{n-1} 100\left(x_{i+1}-x_{i}^{2}\right)^{2}+\left(x_{i}-1\right)^{2}$ & $\mathbf{0}$ & $\mathbf{3 0}$ & $(-100,100)^{n}$ \\
& & & & \\
Griewank & $f_{5}(x)=\frac{1}{4000} \sum_{i=1}^{n} x_{i}^{2}+\prod_{i=1}^{n} \cos \left(\frac{x_{i}}{\sqrt{i}}\right)+1$ & $\mathbf{0}$ & $\mathbf{3 0}$ & $(-600,600)^{n}$ \\
\hline
\end{tabular}


For each benchmark function, all algorithms are independently run 50 times. The solution quality with the optimal value and mean value and optimization rate are reported in Table. 3.

Table 3. The Experiment Results

\begin{tabular}{|c|c|c|c|c|c|}
\hline Function & Opt. & Algorithm & Optimal value & Mean value & Optimization rate \\
\hline & & PSO & $3.580245 \mathrm{E}-008$ & 1.068 253E-006 & $16 \%$ \\
\hline \multirow[t]{3}{*}{ Rastrigrin } & $\mathbf{0}$ & SAPSO & 8.352 534E-030 & $4.681685 \mathrm{E}-028$ & $57 \%$ \\
\hline & & ISAMPSO & $5.566083 \mathrm{E}-038$ & 6.350 471E-036 & $62 \%$ \\
\hline & & PSO & $2.780467 \mathrm{E}-004$ & 5.183 503E-003 & $10 \%$ \\
\hline \multirow[t]{3}{*}{ Schaffer's f6 } & $\mathbf{0}$ & SAPSO & 6.903 523E-027 & 3.640 489E-025 & $95 \%$ \\
\hline & & ISAMPSO & 3.340 436E-031 & 6.814 609E-030 & $100 \%$ \\
\hline & & PSO & 3.403 257E-014 & 4.253 402E-013 & $86 \%$ \\
\hline \multirow[t]{3}{*}{ Sphere } & $\mathbf{0}$ & SAPSO & $5.057492 \mathrm{E}-044$ & 3.471 071E-041 & $93 \%$ \\
\hline & & ISAMPSO & $2.768504 \mathrm{E}-050$ & 8.671 650E-046 & $100 \%$ \\
\hline & & PSO & 1.463 467E-003 & 5.052 562E-003 & $5 \%$ \\
\hline \multirow[t]{3}{*}{ Rosenbrock } & $\mathbf{0}$ & SAPSO & 4.523 708E-004 & 2.562 052E-003 & $78 \%$ \\
\hline & & ISAMPSO & $5.056682 \mathrm{E}-005$ & 2.351 491E-004 & $86 \%$ \\
\hline & & PSO & 7.527 803E-004 & $2.541960 \mathrm{E}-003$ & $88 \%$ \\
\hline \multirow[t]{2}{*}{ Griewank } & $\mathbf{0}$ & SAPSO & 3.602 792E-008 & 6.046 251E-007 & $86 \%$ \\
\hline & & ISAMPSO & $4.131064 \mathrm{E}-010$ & 1.053 592E-008 & $96 \%$ \\
\hline
\end{tabular}

As can be seen in Table.3, the proposed ISAMPSO algorithm can obtain better optimization results than the PSO algorithm and SAPSO algorithm for Rastrigrin, Schaffer's f6, Sphere, Rosenbrock and Griewank. And the obtained optimal solution is very close to the global optimal value for Sphere, Rastrigrin and Schaffer's f6. And for the performance of optimization rate, the proposed ISAMPSO algorithm can obtain higher optimization rate than the PSO algorithm and SAPSO algorithm for all given benchmark functions. For Schaffer's f6 and Sphere functions, the optimization rates of the proposed ISAMPSO algorithm are $100 \%$. So the proposed ISAMPSO algorithm significantly outperforms the PSO algorithm and SAPSO algorithm for all given benchmark functions. In a word, the proposed ISAMPSO algorithm can offer the higher solving accuracy than the PSO algorithm and SAPSO algorithm for all given test functions.

\section{Conclusion}

In allusion to the unbalanced global and local search ability, slow convergence speed and solving accuracy of the PSO algorithm, an improved simulated annealing (ISAM) algorithm based on mutation operator is introduced into the PSO algorithm with crossover and Gauss mutation to propose an improved PSO (ISAMPSO) algorithm in this paper. Because the mutation operator is the most important methods to guide the search for climbing mountain and preventing the convergence, it is introduced into the SA algorithm as a generation mechanism of new solution in 
order to propose an improved simulated annealing (ISAM) algorithm, which is introduced into the PSO algorithm to jump out the local optimum, effectively achieve the global optimum adjust and optimize the population, maintain the diversity of the population, improve the local search ability and convergence speed. Several benchmark functions (Rastrigrin, Schaffer's f6, Sphere, Rosenbrock, Griewank) are selected to test the optimization performance of the proposed ISAMPSO algorithm for solving complex optimization problem. The experiment results show that the proposed ISAMPSO algorithm can effectively overcomes the stagnation phenomenon and enhance the global search ability.

\section{Acknowledgements}

This research was supported by the National Natural Science Foundation of China under Grant No. 61170102, the Natural Science Fund of Hunan Province in China under Grant No. 2015JJ2046, the Scientific Research Fund of Hunan Provincial Education Department under Grant No. 13C030. The program for the initialization, study, training, and simulation of the proposed algorithm in this article was written with the tool-box of MATLAB 2012b produced by the Math-Works.

\section{References}

[1] J. Kennedy and R. C. Eberhart, "Particle swarm optimization", In Proceedings of IEEE International Conference on Neural Networks, Perth, WA, Australia, (1995), 1942-1948.

[2] A. C. Carlos, "Theoretical and numerical constraint handling techniques used with evolutionary algorithms: a survey of the state of the art", Computer Methods in Applied Mechanics and Engineering, vol. 191, no. 11, (2002), pp. 1245-1287.

[3] G. H. Zhang, X. Y. Shao and P. G. Li, "An effective hybrid particle swarm optimization algorithm for multi-objective flexible job-shop scheduling problem", Computers and Industrial Engineering, vol. 56, no. 4, (2009), pp.1309-1318.

[4] A. Jamili, M. Shafia and M. Tavakkoli, "A hybrid algorithm based on particle swarm optimization and simulated annealing for a periodic job shop scheduling problem", International Journal of Advanced Manufacturing Technology, vol. 54, no. 1-4, (2011), pp. 309-322.

[5] G. Moslehi and M. Mahnam, "A Pareto approach to multi-objective flexible job-shop scheduling problem using particle swarm optimization and local search", International Journal of Production Economics, vol. 129, no. 1, (2011), pp. 14-22.

[6] X. H. Shi, Y. C. Liang, H. P. Lee, C. Lu and L. M. Wang, "An improved GA and a novel PSO-GAbased hybrid algorithm”, Information Processing Letters, vol. 93, no. 5, (2005), pp. 255-261.

[7] Q. Tang, Y. W. Wu, X. Y. Xiong and S. H. Lou, "Generation expansion planning of hydro-thermal power system based on SA-PSO”, High Voltage Engineering, vol. 32, no. 4, (2005), pp. 104-107.

[8] D. H. Kim and K. Hirota, "Vector control for loss minimization of induction motor using GA-PSO", Applied Soft Computing Journal, vol. 8, no. 4, (2008), pp. 1692-1702.

[9] W. T. Li, X. W. Shi, L. Xu and Y. Q. Hei, "Improved GA and PSO culled hybrid algorithm for antenna array pattern synthesis", Progress in Electromagnetics Research, vol. 80, (2008), pp. 461-476.

[10] T. Niknam, B. Amiri, J. Olamaei and A. Arefi, "An efficient hybrid evolutionary optimization algorithm based on PSO and SA for clustering", Journal of Zhejiang University: Science A, vol. 10, no. 4, (2009), pp. 512-519.

[11] S. C. Neoh, N. Morad, C. P. Lim and Z. A. Aziz, "A GA-PSO layered encoding evolutionary approach to 0/1 knapsack optimization", International Journal of Innovative Computing, Information and Control, vol. 6 , no. 8, (2010), pp. 3489-3505.

[12] J. Behnamian and S. M. T. Fatemi Ghomi, "Development of a PSO-SA hybrid metaheuristic for a new comprehensive regression model to time-series forecasting", Expert Systems with Applications, vol. 37, no. 2, (2010), pp. 974-984.

[13] W. P. Lee and Y. T. Hsiao, "An adaptive GA - PSO approach with gene clustering to infer S-system models of gene regulatory networks", Computer Journal, vol. 54, no. 9, (2011), pp. 1449-1464.

[14] K. Thanushkodi and K. Deeba, "On performance analysis of hybrid algorithm (Improved PSO with simulated annealing) with GA, PSO for multiprocessor job scheduling", WSEAS Transactions on Computers, vol. 10, no. 9, (2011), pp. 287-300.

[15] M. A. Rahim, H. M. Khalid and A. Khoukhi, "Nonlinear constrained optimal control problem: A PSOGA-based discrete augmented Lagrangian approach", International Journal of Advanced Manufacturing Technology, vol. 62, no. 1-4, (2012), pp. 183-203. 
[16] S. Soleymani, "Bidding strategy of generation companies using PSO combined with SA method in the pay as bid markets", International Journal of Electrical Power and Energy Systems, vol. 33, no. 7, (2011), pp. 1272-1278.

[17] A. Tajbakhsh, K. Eshghi and A. Shamsi, "A hybrid PSO-SA algorithm for the traveling tournament problem", European Journal of Industrial Engineering, vol. 6, no. 1, (2012), pp. 2-25.

[18] S. G. Wang, F. J. Wang and X. F. Du, "A method for solving the shortest path on curved surface based on PSO-SA algorithm", Journal of Theoretical and Applied Information Technology, vol. 46, no. 2, (2012), pp. 672-676.

[19] Y. Z. Lee and S. G. Ponnambalam, "Optimization of multi-pass turning operations using PSO and GAAIS algorithms", International Journal of Production Research, vol. 50, no. 22, (2012), pp. 6499-6518.

[20] M. Sheikhalishahi, V. Ebrahimipour, H. Shiri, H. Zaman and M. Jeihoonian, "A hybrid GA-PSO approach for reliability optimization in redundancy allocation problem", International Journal of Advanced Manufacturing Technology, vol. 68, no. 1-4, (2013), pp. 317-338.

[21] A. Galvez and A. Iglesias, "A new iterative mutually coupled hybrid GA-PSO approach for curve fitting in manufacturing", Applied Soft Computing Journal, vol. 13, no. 3, (2013), pp. 1491-1504.

[22] I. Pan and S. Das, "Design of hybrid regrouping PSO-GA based sub-optimal networked control system with random packet losses", Mimetic Computing, vol. 5, no. 2, (2013), pp. 141-153.

[23] M. Rabbani, M. Baghersad and R. Jafari, "A new hybrid GA-PSO method for solving multi-period inventory routing problem with considering financial decisions", Journal of Industrial Engineering and Management, vol. 6, no. 4, (2013), pp. 909-929.

[24] S. G. Wang, F. F. Zhou and F. J. Wang, "Effect of inertia weight $\omega$ on PSO-SA algorithm", International Journal of Online Engineering, vol. 9, no. 6, (2013), pp. 87-91.

[25] F. Khoshahval, A. Zolfaghari, H. Minuchehr and M. R. Abbasi, "A new hybrid method for multiobjective fuel management optimization using parallel PSO-SA", Progress in Nuclear Energy, vol. 76, (2014), pp. 112-121.

[26] R. Martínez-Soto, O. Castillo and L. T. Aguilar, "Type-1 and Type-2 fuzzy logic controller design using a Hybrid PSO-GA optimization method", Information Sciences, vol. 285, no. 1, (2014), pp. 35-49.

[27] L. Sahoo, A. Banerjee, A. K. Bhunia and S. Chattopadhyay, "An efficient GA-PSO approach for solving mixed-integer nonlinear programming problem in reliability optimization", Swarm and Evolutionary Computation, vol. 19, (2014), pp. 43-51.

[28] V. Sarasvathi, N. C. S. N. Iyengar and S. Saha, "QoS guaranteed intelligent routing using hybrid PSOGA in wireless mesh networks", Cybernetics and Information Technologies, vol. 15, no. 1, (2015), pp. 69-83.

[29] M. Tao, S. Q. Huang, Y. Li, M. Yan and Y. Y. Zhou, "SA-PSO based optimizing reader deployment in large-scale RFID Systems", Journal of Network and Computer Applications, vol. 52, (2015), pp. 90-100.

[30] S. Kirkpatric, J. C. D. Gelatt and M. P. Vecchi, "Optimization by simulated annealing", Science, vol. 220, no. 4598, (1983), pp. 671-680.

\section{Authors}

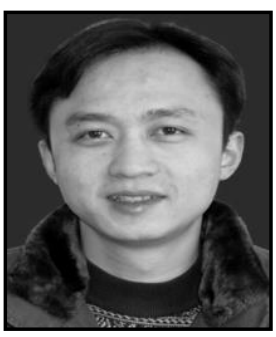

Xiaojun Deng, $\mathrm{He}$ is an assistant professor in the school of computer and communication, Hunan University of Technology. In 2004, he received the master degree in computer science and Technology from National University of Defense Technology, Changsha, China. His research interests include image processing and computer network.

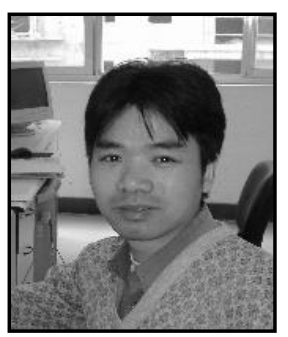

Zhiqiang Wen, He is currently a Professor with the School of Computer and Communication, Hunan University of Technology, Zhuzhou, China. He received the B.E. degree from the Central South University of Technology, Changsha, China, in 1997, the M.S. degree in computer application technology from Guangxi University, Nanning, China, in 2004, and the Ph.D. degree in computer application technology from Central South University, Changsha, in 2008. He is a member of the China Computer Federation. His research interests include computer vision, digital image processing, and robotics. 


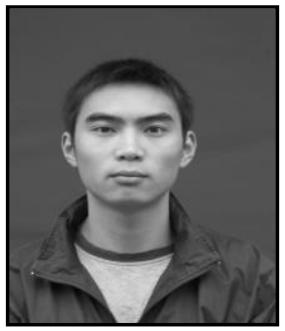

Yu Wang, He is a lecturer in the school of computer and communication, Hunan University of Technology. In 2014 he received the master degree in computer science and Technology from Hunan University of Technology Zhu Zhou, China. His research interests include image processing and Context Awareness.

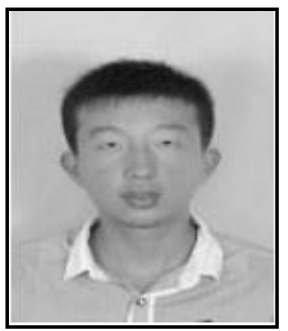

Pingan Xiang, He is a college student in the school of computer and communication, Hunan University of Technology. His research interests include software development. 
International Journal of Multimedia and Ubiquitous Engineering

Vol.10, No.10 (2015) 